ISSN 0103-9954

\title{
APORTE E DECOMPOSIÇÃO DA SERAPILHEIRA NA FLORESTA ATLÂNTICA, ILHA DA MARAMBAIA, MANGARATIBA, RJ
}

\author{
LITTER DEPOSITION AND DECOMPOSITION IN A FRAGMENT OF ATLANTIC FOREST IN THE \\ ISLAND OF MARAMBAIA, MANGARATIBA, RJ, BRAZIL
}

\author{
Marcos Gervasio Pereira $^{1}$ Luis Fernando Tavares de Menezes ${ }^{2}$ Nivaldo Schultz ${ }^{3}$
}

\section{RESUMO}

A produção e a decomposição de serapilheira de um fragmento de Floresta Atlântica de encosta na Ilha da Marambaia, Mangaratiba, RJ, foram estudadas durante o período de dezembro de 2003 a novembro de 2004. O aporte de serapilheira foi mensurado partindo de trinta coletores cônicos. A taxa de decomposição da serapilheira foi quantificada por meio de 36 sacolas de decomposição instaladas na área. Para avaliação da fertilidade do solo foram coletadas amostras nas profundidades de 0-5, 5-10 e 10-20 cm. A produção total de serapilheira foi de $7,9 \mathrm{Mg} \mathrm{ha}^{-1}$, sendo a maior deposição verificada no mês de novembro e a menor em junho. A fração foliar foi a de maior contribuição em relação às demais. A taxa de decomposição da serapilheira foi $40 \%$ em 7 meses de observação, seguindo modelo exponencial. Verificou-se comportamento diferenciado quanto à liberação de N, P e K. Com exceção do fósforo, os maiores valores de nutrientes, carbono e $\mathrm{H}+\mathrm{Al}$ foram observados na camada superficial.

Palavras-chave: ciclagem de nutrientes; decomposição; florestas tropicais.

\section{ABSTRACT}

Litter production and decomposition of an Atlantic Forest fragment in Marambaia Island, Mangaratiba, RJ, were monitored from December 2003 to November 2004. For the litter deposition evaluation, 30 litter traps were installed and 36 litter bags were allocated in the area to quantify litter decomposition. Soil samples were collected at $0-5 \mathrm{~cm}, 5-10 \mathrm{~cm}$ and $10-20 \mathrm{~cm}$ depth to evaluate soil fertility. The litter production was $7.9 \mathrm{Mg} \mathrm{ha}^{-1}$ and the highest deposition was verified in November and the lowest in June. The leaf fraction presented the highest contribution comparing to the others. In seven months of observation, litter decomposition rate was $40 \%$ and showed an exponential decrease. Different behavior for $\mathrm{N}, \mathrm{P}$ and $\mathrm{K}$ release was verified. Exception for $\mathrm{P}$, carbon, nutrients and $\mathrm{H}+\mathrm{Al}$ presented highest concentrations in superficial layer.

Keywords: nutrient cycling; decomposition; tropical forests.

\section{INTRODUÇÃO}

Nos ecossistemas florestais, as reservas minerais e orgânicas acumulam-se na biomassa vegetal, animal, na serapilheira e no solo. Este último é um importante compartimento, de onde é retirada a maioria dos minerais necessários à manutenção das plantas (BRITEZ et al., 1997; PAGANO e DURIGAN, 2000). O aporte de serapilheira e sua subseqüente decomposição compreendem processos de grande relevância para a manutenção da fertilidade do solo, constituindo importantes etapas da ciclagem de nutrientes dentro dos ecossistemas (KRAMMER e KOZLOWKI, 1960; FASSEBENDER,1993; GAMA-RODRIGUES, 1997; BARBOSA, 2000). A deposição da serapilheira pode variar de acordo o estádio sucessional da formação florestal (DELITTI, 1989), grau de perturbação da área (MARTINS e RODRIGUES, 1999) e do tipo de vegetação considerado.

A quantificação da produção de serapilheira tem contribuído para a compreensão do funcionamento e manejo de florestas, particularmente nas regiões tropicais (JORDAN, 1985; DELITTI, 1995). Assim, vários autores têm investigado a produção de serapilheira e sua decomposição em diferentes formações florestais contribuindo para um maior conhecimento da ciclagem de nutrientes dentro dos ecossistemas (WERNECK et al., 2001; BACKES et al., 2004; TOLEDO e PEREIRA, 2004).

1. Engenheiro Florestal, Dr., Professor Associado II do Departamento de Solos, Universidade Federal Rural do Rio de Janeiro, BR 465 Km 7, CEP 23890-000, Seropédica (RJ). Bolsista do CNPq. gervasio@ufrrj.br

2. Biólogo, Dr., Professor Adjunto da Universidade Federal do Espírito Santo, Centro Universitário Norte do Espírito Santo. Rua Humberto de Almeida Fanklin 257, Bairro Centro, CEP 29933-415, São Mateus (ES).

3. Engenheiro Agrônomo, Acadêmico de Mestrado do Curso de Pós-Graduação em Agronomia, Universidade Federal Rural do Rio de Janeiro, BR 465 Km 7, CEP 23890-000, Seropédica (RJ). Bolsista do CNPq.

Recebido para publicação em 5/07/2006 e aceito em 7/10/2008. 
As taxas de decomposição da serapilheira e a liberação dos nutrientes em ecossistemas tropicais são consideradas altas, em conseqüência das elevadas taxas de precipitação e temperatura (ALVAREZ-SÁNCHES e ENRÍQUEZ, 1996). Aliada a composição química das diferentes frações que compõem a serapilheira (destacadamente as folhas), tem-se sugerido que os principais fatores de controle da decomposição são a temperatura e a precipitação (DICKINSON, 1974; JENSEN 1974). Além disso, a qualidade do substrato e a fauna do solo podem ser cruciais para a decomposição em florestas tropicais (LA CARO e RUDD, 1985; BLOOMFIELD et al., 1993; CORTEZ et al., 1996; GONZÁLEZ e SEASTEDT, 2001).

O processo de decomposição da serapilheira libera parte do carbono acumulado na biomassa, sendo que uma parte do carbono retorna para a atmosfera como $\mathrm{CO}_{2}$ e os outros elementos assumem uma forma novamente utilizável pelas plantas (STEVENSON, 1982). Esse ciclo é constantemente renovado por um conjunto de processos interconectados de forma que os mesmos recursos nutricionais são utilizados sucessivamente na fixação de energia (DELITTI, 1995).

O conhecimento das taxas de produção e decomposição da serapilheira, nas fisionomias que compõem o bioma Mata Atlântica, é de fundamental importância, visto o grau de antropização em que ele se encontra. Por meio dessas avaliações, pode-se obter um conjunto de informações que podem contribuir para um melhor conhecimento do bioma e funcionar também como ferramenta no planejamento do manejo a ser adotado (GARAY e KINDEL, 2001). Neste sentido, este estudo teve como objetivo avaliar o aporte de serapilheira e a decomposição do material decíduo de um trecho de floresta atlântica na Ilha da Marambaia, RJ.

\section{MATERIAL E MÉTODOS}

\section{Localização e características da área de estudo}

A Ilha da Marambaia se localiza na baía de Sepetiba (Figura 1), município de Mangaratiba, sul do estado do Rio de Janeiro ( $23^{\circ} 04^{\prime} \mathrm{S}$ e $43^{\circ} 53^{\prime} \mathrm{W}$ ) e compõe o alinhamento geológico formado pelas ilhas da Madeira, Itacuruçá e Jaguanum. Seu pico mais alto atinge $641 \mathrm{~m}$ de altura e sua área florestada é de 2.125,43 ha (CONDE et al., 2005), caracterizada pela floresta ombrófila densa submontana (IBGE, 1992). A leste da Ilha, ancora-se uma extensa faixa arenosa, com cerca de $40 \mathrm{~km}$ de comprimento, conhecida como restinga da Marambaia. A floresta atlântica na Ilha da Marambaia, embora tenha sido submetida a diversas interferências humanas no passado (lavouras de café, entreposto de escravos, escola de pesca e indústria de conserva de pescado e por décadas abriga uma comunidade de caiçaras) Conde et al. (2005) constitui um importante remanescente de floresta no estado do Rio de Janeiro.

Este estudo foi realizado na vertente noroeste da Ilha, voltada para a Baía de Sepetiba onde a vegetação não é submetida à intervenções humanas desde o inicio da década de 1980, quando se instalou na região o Centro de Adestramento da Ilha da Marambaia - Marinha do Brasil.

$\mathrm{Na}$ área de estudo foram quantificadas 42 famílias e 128 espécies florestais, sendo que destas as que apresentaram a maior densidade absoluta e relativa, freqüência absoluta e relativa e IVI (índice de valor de importância) foram: Guapira opposita (Vell.) Reitz, Vochysia oppugnata (Vell.) Warm, Nectandra oppositifolia Nees \& Mart, Pseudopiptaenia contorta (DC.) G.P. Lewis \& M. P. Lima, Xylopia sericea A. St.-Hill, Tabernaemontana latae Miers, Myrcia splends (SW.) DC., Cybistax antissiphylitica Mart, Ocotea schotti (Meisn.) Mez e Inga laurina (Sw.) Willd. Quanto ao estádio sucessional, na área de estudo, verificase o predomínio de espécies secundárias iniciais e tardias. Uma caracterização dos aspectos florísticos e vegetacionais da floresta atlântica da Marambaia encontra-se em Conde et al. (2005). 


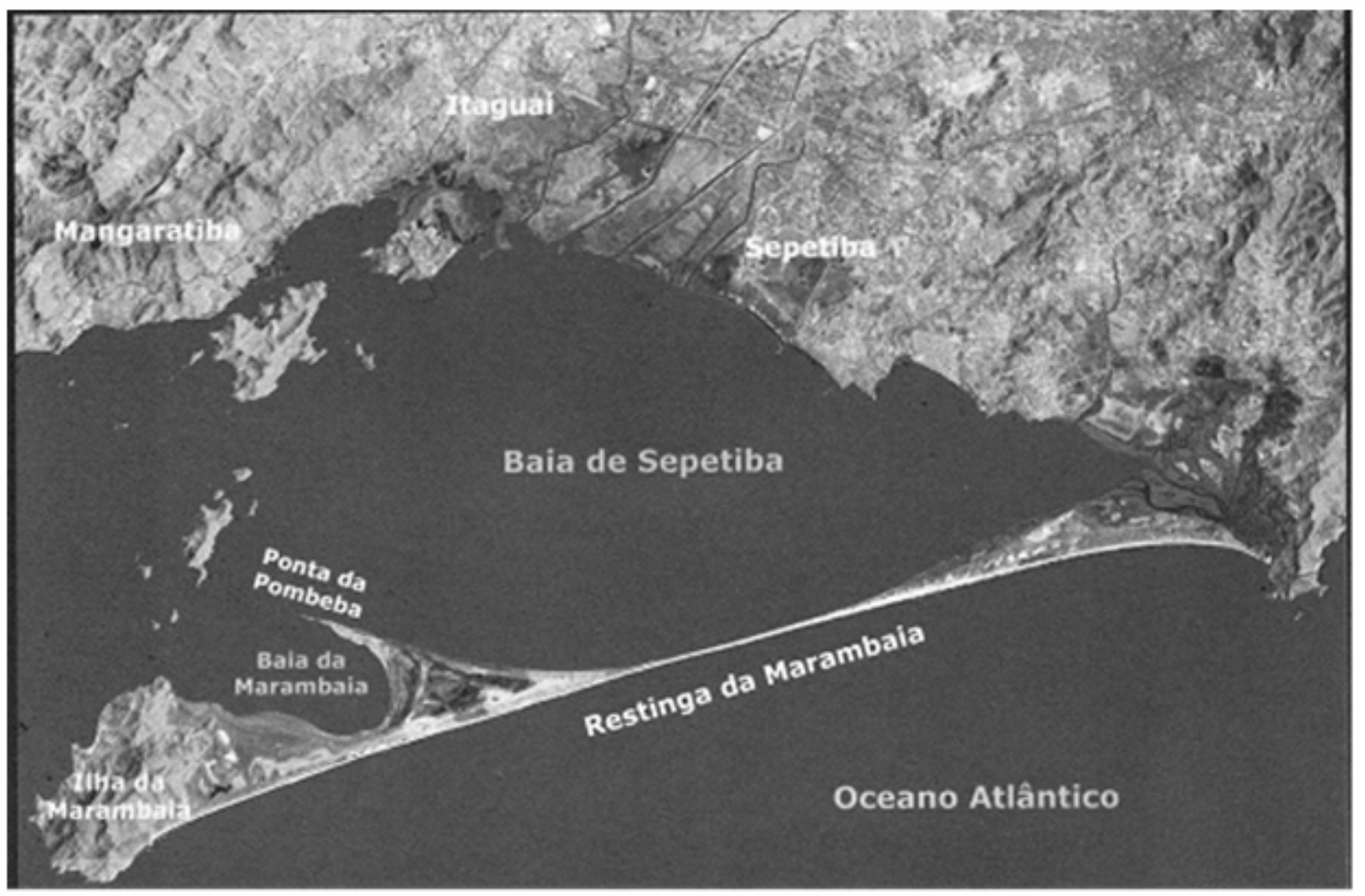

FIGURA 1: Localização da Restinga da Marambaia no estado do Rio De Janeiro. (Fonte: Menezes, 2005). FIGURE 1: Restinga da Marambaia location, in Rio de Janeiro, Brazil. (Menezes, 2005).

Os dados climatológicos foram obtidos no posto meteorológico localizado na restinga da Marambaia, a $9,7 \mathrm{~m}$ de altitude e a $50 \mathrm{~m}$ do mar, distando $42 \mathrm{~km}$ da área de estudo. $\mathrm{O}$ clima da região enquadra-se no macroclima Aw - clima tropical chuvoso, segundo Köppen. As temperaturas do ar da região são típicas das áreas litorâneas tropicais. As médias mensais se situam sempre acima de $20,0^{\circ} \mathrm{C}$ e a média anual alcança $23,7^{\circ} \mathrm{C}$. Em fevereiro, ocorre a maior temperatura média mensal $\left(26,8^{\circ} \mathrm{C}\right)$ e, em agosto, a menor $\left(20,9^{\circ} \mathrm{C}\right)$. A precipitação média anual é de $1239,7 \mathrm{~mm}$, sendo $37 \%$ ocorrendo no verão durante a estação de chuvas e $15 \%$ no inverno, durante a estação mais seca. Em julho e agosto, a precipitação média mensal situa-se entre 40 e $55 \mathrm{~mm}$ e, em novembro e março, os índices pluviométricos são sempre superiores a $100 \mathrm{~mm}$ (MATTOS, 2005).

\section{Quantificação da serapilheira}

Para a quantificação do aporte de serapilheira foram instalados trinta coletores cônicos de área de $0,45 \mathrm{~m}^{2}$, confeccionados com tela de náilon com malha de $1 \mathrm{~mm}$, suspensos do solo a $80 \mathrm{~cm}$. Os coletores foram distribuídos dentro de uma área de 1 ha, distantes entre si com mínimo de $50 \mathrm{~m}$. A coleta do material decíduo depositado nos coletores foi feita mensalmente durante o período de dezembro de 2003 a novembro de 2004, sendo este acondicionado em sacos de papel. No laboratório o material foi seco ao ar e posteriormente separado nas frações folhas, galhos (até $2 \mathrm{~cm}$ de diâmetro), frutos, flores e resíduos (material não identificável). Após a triagem, o material foi seco em estufa a $65^{\circ} \mathrm{C}$ durante 48 horas, quando atingiu peso constante, pesando-se cada fração separadamente, a fim de se quantificar a sua contribuição. A partir desses dados, foi estimada a produção de serapilheira no período de estudo, com base na equação:

$\mathrm{PS}=(\Sigma \mathrm{PM} \times 10.000) / \mathrm{Ac}$

Em que: $\mathrm{PS}=$ Produção de serapilheira $\left(\mathrm{kg} \mathrm{ha}^{-1} \mathrm{ano}^{-1}\right) ; \mathrm{PM}=$ Produção mensal de serapilheira $\left(\mathrm{kg} \mathrm{ha}^{-1} \mathrm{mês}^{-1}\right)$; $\mathrm{Ac}=$ Área do coletor $\left(\mathrm{m}^{2}\right)$.

Depois de pesado, o material foi homogeneizado, moído em moinho do tipo Willey e submetido à digestão sulfúrica (TEDESCO et al., 1995). No extrato obtido foram determinados os teores de nitrogênio $(\mathrm{N})$, fósforo (P) e potássio (K) (TEDESCO et al., 1995). 


\section{Avaliação da decomposição da serapilheira (fração foliar) e liberação de nutrientes}

Para avaliar a decomposição da serapilheira, foram utilizados 36 sacos de decomposição de dimensões de $25 \times 25 \mathrm{~cm}$ e malha de $4 \mathrm{~mm}$ distribuídos próximos aos coletores. Em cada saco foram acondicionados $10 \mathrm{~g}$ de folhas, que haviam sido depositadas nos coletores cônicos. As coletas dos sacos de decomposição foram realizadas aos $30,90,150$ e 210 dias. A cada coleta eram retirados três sacos de decomposição da área de estudo. $\mathrm{O}$ material foi seco em estufa a $65^{\circ} \mathrm{C}$ durante 48 horas, sendo posteriormente pesado. Após pesagem, o material de cada coleta foi homogeneizado, moído em moinho do tipo Willey e submetido à digestão sulfúrica (TEDESCO et al., 1995). No extrato obtido foram determinados os teores de nitrogênio (N), fósforo (P) e potássio (K) (TEDESCO et al., 1995).

A determinação da taxa de decomposição foi realizada por meio de medidas de perda de massa do material contido nas sacolas. De posse dos valores de perda de massa ao longo do período de estudo, foi estimada a constante de decomposição k, conforme Thomas e Asakawa (1993), usando o modelo exponencial apresentado: $X_{t}=X_{0} \cdot e^{-k t}$; onde $X_{t}$ é o peso do material remanescente após $t$ dias e $X_{0}$ o peso do material seco originalmente colocado nos sacos no tempo zero $(\mathrm{t}=0)$. O coeficiente desse modelo exponencial, bem como as curvas que caracterizam a perda de peso (decomposição) de cada resíduo, foram confeccionados com o auxílio do programa SIGMAPLOT. O tempo de meia-vida $\left(\mathrm{T}^{1 / 2}\right)$ desse resíduo foi calculado conforme Rezende et al. (1999) por meio da equação: $\mathrm{T}^{1 / 2}=\ln (2) / \mathrm{k}$ em que $\mathrm{k}$ é a constante de decomposição estimada pelo programa citado anteriormente. Esse modelo também foi utilizado para descrever a liberação dos nutrientes, sua constante de decomposição $(\mathrm{k})$ e tempo de meia-vida $\left(\mathrm{T}^{1 / 2}\right)$.

\section{Avaliação da fertilidade do solo}

Para avaliação da fertilidade do solo, foram coletadas três amostras compostas, em uma área de 1 ha, nas profundidades de 0 a 5,5 a 10 e 10 a $20 \mathrm{~cm}$, sendo cada amostra composta formada por dez amostras simples. Nas amostras, foram quantificados os teores de carbono orgânico, $\mathrm{pH}, \mathrm{N}, \mathrm{P}, \mathrm{K}^{+}, \mathrm{Ca}^{2+}$, $\mathrm{Mg}^{2+}, \mathrm{Al}^{3+}, \mathrm{Na}^{+}$e $\mathrm{H}+\mathrm{Al}$, segundo EMBRAPA (1997).

As análises estatísticas foram realizadas com o emprego do pacote estatístico SAEG 5.0 (Sistema de Análises Estatísticas e Genéticas - Universidade Federal de Viçosa)

\section{RESULTADOS E DISCUSSÃO}

\section{Produção de serapilheira}

A produção total de serapilheira durante o período estudado foi de $7,9 \mathrm{Mg} \mathrm{ha}^{-1}$, sendo que as maiores e menores deposições foram verificadas nos meses de novembro e junho $\left(1,3 \mathrm{Mg} \mathrm{ha}^{-1}\right.$ e $\left.0,3 \mathrm{Mg} \mathrm{ha}^{-1}\right)$ respectivamente (Figura 2). A produção de serapilheira estimada neste estudo se enquadra na prevista para florestas situadas em latitudes de $25^{\circ}$, valores entre 7,0 a 8,8 $\mathrm{Mg} \mathrm{ha}^{-1}$ ano $^{-1}$ (BRAY e GORHAM, 1964).

Os valores observados são superiores aos reportados por Pires et al. (2006) em que os autores quantificando a deposição de serapilheira em uma restinga da Ilha do Mel (PR) verificaram um aporte de 5,1 $\mathrm{Mg} \mathrm{ha}^{-1}$, atribuindo esses baixos valores às condições oligotróficas do ambiente. Baixos valores de deposição associados a condições de oligotrofismo também foram verificados nos trabalhos de GARAY e KINDEL (2001) e BRITEZ et al. (2005).

Não foram verificadas correlações entre a deposição de serapilheria e as taxas de precipitação $\left(0,38^{\text {ns }}\right)$, sendo os maiores valores observados nos meses de outubro e novembro. Alguns estudos em Floresta Atlântica indicam maior produtividade de serapilheira acompanhando os períodos de maior pluviosidade (MEGURO et al., 1979; VARJABEDIAN e PAGANO, 1988; MORAES et al., 1999, PIRES et al., 2006), apesar de um maior número de trabalhos reportar maiores deposições de serapilheira na época seca (CUEVAS e MEDINA, 1988; LOUZADA, et al.,1995; VILLELA e PROCTOR, 2002). Maior produção de serapilheira coincidindo com períodos chuvosos também tem sido constatada para outros sistemas florestais no Brasil (JACKSON, 1978; MORAES et al., 1999; CARMO e MORELLATO, 2000; VITAL et al., 2004) e no mundo (HERBOHN e CONGDON, 1993). 


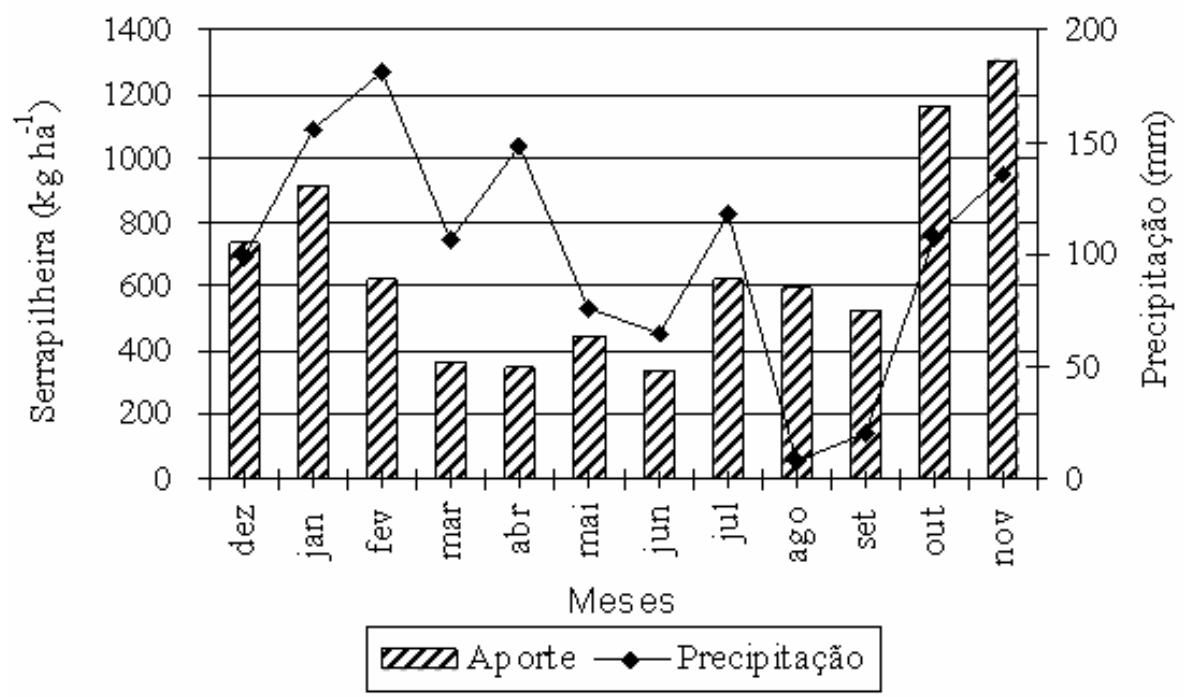

FIGURA 2: Aporte de serapilheira e precipitação na floresta atlântica da Ilha da Marambaia, RJ.

FIGURE 2: Litter contribution and rainfall in the Atlantic forest of the Island of Marambaia, RJ.

Quanto à composição da serapilheira verificou-se uma elevada participação da fração folhas $(66 \%)$, seguida da fração galhos (21\%), resíduos (8\%), frutos (4\%) e flores (1\%) (Figura 3). Estudos realizados na mata atlântica e em outros sistemas florestais, normalmente relatam uma participação,em torno de $70 \%$, da fração foliar na composição da serapilheira (MEGURO et al., 1979; PAGANO, 1989; MORELLATO, 1992; LOUZADA et al., 1995; FIGUEIREDO FILHO et al., 2003; TOLEDO e PEREIRA, 2004, BACKES et al., 2004). Pires et al. (2006) estudando a produção, acúmulo e decomposição da serapilheira em uma restinga da Ilha do Mel (PR) verificaram que a fração foliar contribuiu com $(74,6 \%)$ do total da serapilheira aportada.

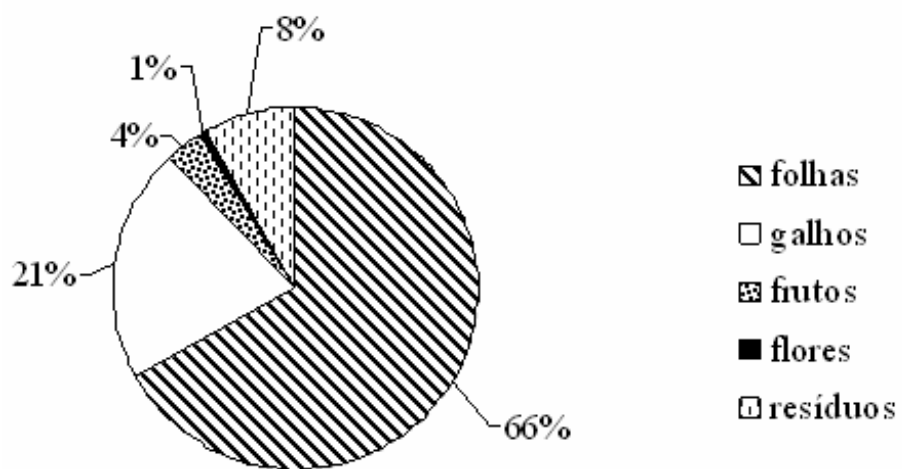

FIGURA 3: Distribuição das frações constituintes da serapilheira na floresta atlântica da Ilha da Marambaia, RJ.

FIGURE 3: Litter constituent fractions distribution in the Atlantic forest of the Island of Marambaia, RJ.

\section{Conteúdo de nutrientes na serapilheira}

A deposição mensal dos nutrientes é apresentada no Tabela 1. O retorno anual de nutrientes foi de $121,99 \mathrm{~kg}$ de N, 2,01 kg de P e 32,08 kg de K. Resultados similares foram encontrados por Moraes et al. (1999), em Floresta Atlântica na Ilha do Cardoso, SP, onde o retorno de nutrientes foi estimado em 101,8 kg de N; 3,8 kg de P e 20,3 kg de K. Em floresta semidecidual em São Paulo, localizada em zona ripária, Vital et al., (2004), verificaram valores mais elevados, com deposição de nutrientes de $217,76 \mathrm{~kg} \mathrm{ha}^{-1} \mathrm{de} \mathrm{N} ; 11,55$ $\mathrm{kg} \mathrm{ha}^{-1}$ de P e $52,79 \mathrm{~kg} \mathrm{ha}^{-1}$ de $\mathrm{K}$

Foram constadas variações temporais para os nutrientes estudados, havendo uma tendência de maior adição de nutrientes nos meses de maior aporte de serapilheira (outubro, novembro, dezembro e janeiro). Vital et al. (2004) observaram variações temporais na deposição de nutrientes, constatando maiores variações para o $\mathrm{K} \mathrm{e} \mathrm{o} \mathrm{Mg.}$ 
TABELA 1: Conteúdo de N, P e K na serapilheira.

TABLE 1: Concentration of N, P and $\mathrm{K}$ in the litter.

\begin{tabular}{|c|c|c|c|c|}
\hline \multirow{2}{*}{\multicolumn{2}{|c|}{ Mês }} & $\mathrm{N}$ & $P$ & $\mathrm{~K}$ \\
\hline & & \multicolumn{3}{|c|}{$\mathrm{g} \mathrm{kg}^{-1}$} \\
\hline \multirow{12}{*}{ 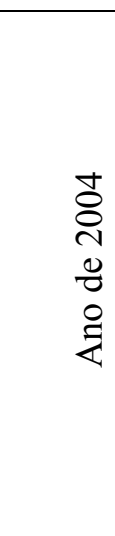 } & Dezembro/2003 & 9,30 & 0,16 & 2,15 \\
\hline & Janeiro & 11,64 & 0,17 & 2,10 \\
\hline & Fevereiro & 7,61 & 0,13 & 2,52 \\
\hline & Março & 5,36 & 0,09 & 0,90 \\
\hline & Abril & 5,28 & 0,09 & 1,64 \\
\hline & Maio & 6,58 & 0,10 & 1,76 \\
\hline & Junho & 4,80 & 0,09 & 1,09 \\
\hline & Julho & 8,59 & 0,15 & 3,93 \\
\hline & Agosto & 8,26 & 0,13 & 3,69 \\
\hline & Setembro & 7,79 & 0,13 & 3,36 \\
\hline & Outubro & 15,71 & 0,30 & 3,18 \\
\hline & Novembro & 21,69 & 0,47 & 5,76 \\
\hline \multicolumn{2}{|l|}{ Média } & 9,38 & 0,17 & 2,67 \\
\hline \multicolumn{2}{|c|}{ Desvio-Padrão } & 4,92 & 0,11 & 1,38 \\
\hline
\end{tabular}

Decomposição da serapilheira e liberação de nutrientes

A taxa de decomposição foi de $40 \%$ em 7 meses de observação (Figura 4) caracterizando a lenta e constante decomposição do material. O fato da decomposição seguir o modelo exponencial indica que essa não é constante ao longo do tempo. A decomposição está ligada a diversos fatores ambientais, químico e físicos do próprio material Cianciaruso et al. (2006). A constante de decomposição (k) da serapilheira foi baixa $\left(0,0023 \mathrm{~g} \mathrm{~g}^{-1}\right)$ e o tempo de meia-vida $\left(\mathrm{T}^{1 / 2}\right)$ foi de 301 dias. Pires et al. (2005) estudando a decomposição da serapilheira em uma área de restinga na Ilha do Mel (PR) observaram uma lenta decomposição do material aportado, sugerindo como possível explicação para a baixa decomposição uma adaptação do ecossistema para minimizar as perdas por lixiviação e permitir um melhor aproveitamento dos nutrientes. Comportamento similar ao verificado neste estudo foi observado por Cianciaruso et al. (2006) estudando a produção de serapilheira e decomposição do material foliar em um cerradão na Estação Ecológica de Jataí, município de Luiz Antônio, SP. Os autores verificaram lenta decomposição da serapilheira, sendo que ao final de um ano, apenas $43 \%$ da massa original foi decomposta. Apesar de se tratar de um ecossistema diferente do analisado neste estudo, a lenta taxa de decomposição da serapilheira, também foi atribuída às condições de oligotrofismo verificadas na área de cerrado.

Quanto à liberação de nutrientes, observa-se que estes apresentaram comportamento diferenciado. Para o N (Figura 5) verificou-se um comportamento exponencial, sendo a constante de decomposição (k) de 0,0025 , similar a observada para a decomposição da serapilheira, já o $\mathrm{T}^{1 / 2}$ foi de 277 dias. Aidar e Joly (2003), estudando a decomposição da serapilheira de araribá (Centrolobium tomentosum Guill. ex Benth. Fabaceae) em uma mata ciliar, em São Paulo, observaram uma lenta taxa de liberação de N, sendo o tempo necessário para a que ocorresse a liberação de $50 \%$ da quantidade inicial bastante alto (697 dias). No estudo, também foi verificada maior liberação de $\mathrm{N}$ na fase final de decomposição do material decíduo. Esse comportamento pode ser decorrente de um possível processo de tanização de proteínas pelos fenóis produzidos na decomposição ou pela imobilização do N no protoplasma microbiano Swift et al. (1979). 


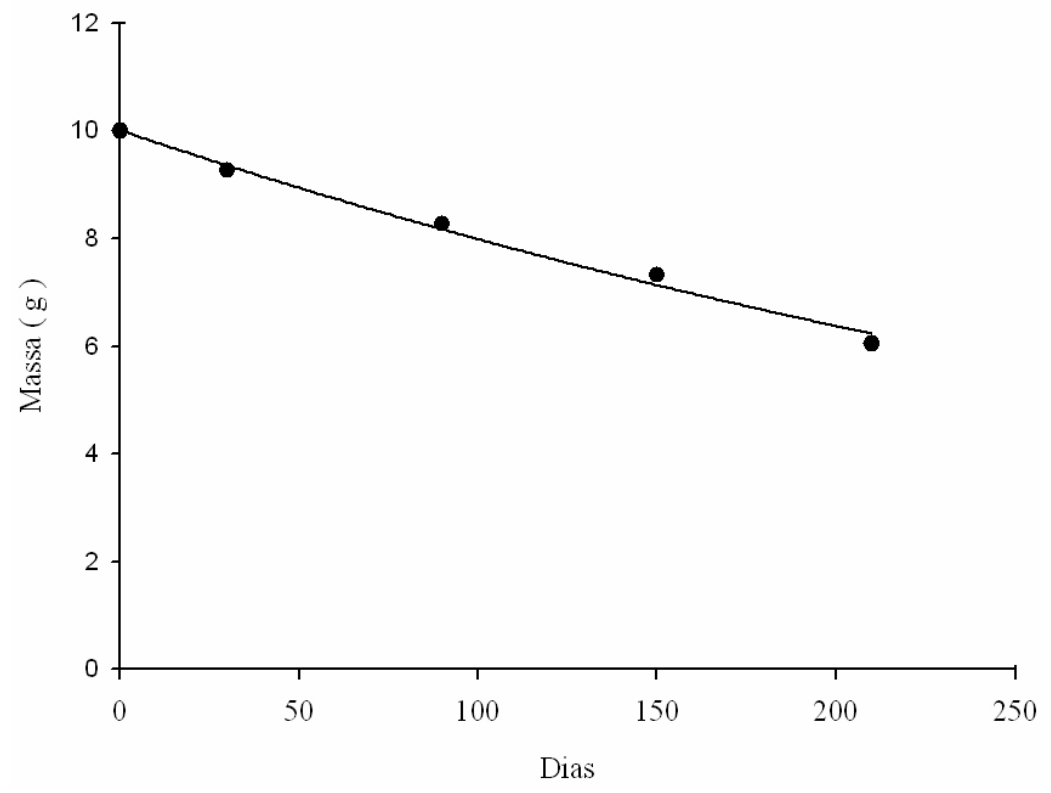

FIGURA 4: Curva de decomposição da serapilheira na floresta atlântica da Ilha da Marambaia, RJ. FIGURE 4: Litter decomposition curve in the Atlantic forest of the Island of Marambaia, RJ.

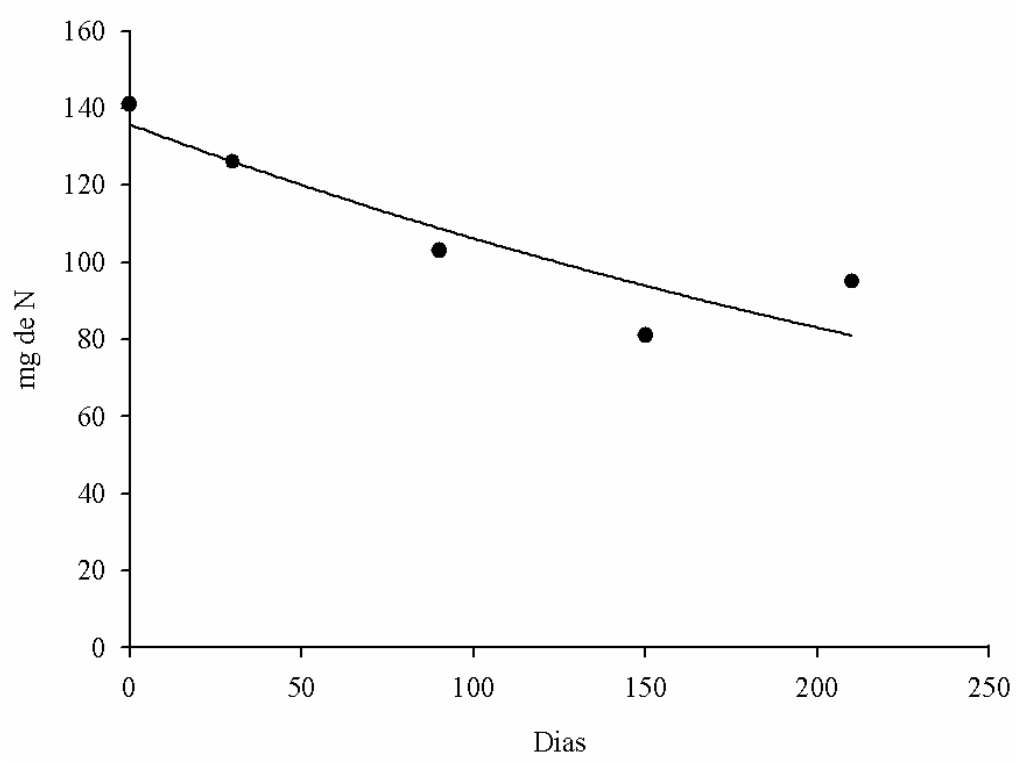

FIGURA 5: Liberação de nitrogênio na floresta atlântica da Ilha da Marambaia, RJ.

FIGURE 5: Nitrogen release in the Atlantic forest of the Island of Marambaia, RJ.

Para o P (Figura 6) foi observada uma liberação irregular desse nutriente no decorrer do tempo, comportamento similar ao verificado por Aidar e Joly (2003) avaliando a decomposição da fração foliar de Centrolobium tomentosum. Foi verificada uma constante de decomposição (k) de $0,0025 \mathrm{~g} \mathrm{~g}^{-1} \mathrm{e} \mathrm{um} \mathrm{T}^{1 / 2}$ de 277 dias, demonstrando a lenta liberação desse nutriente. 


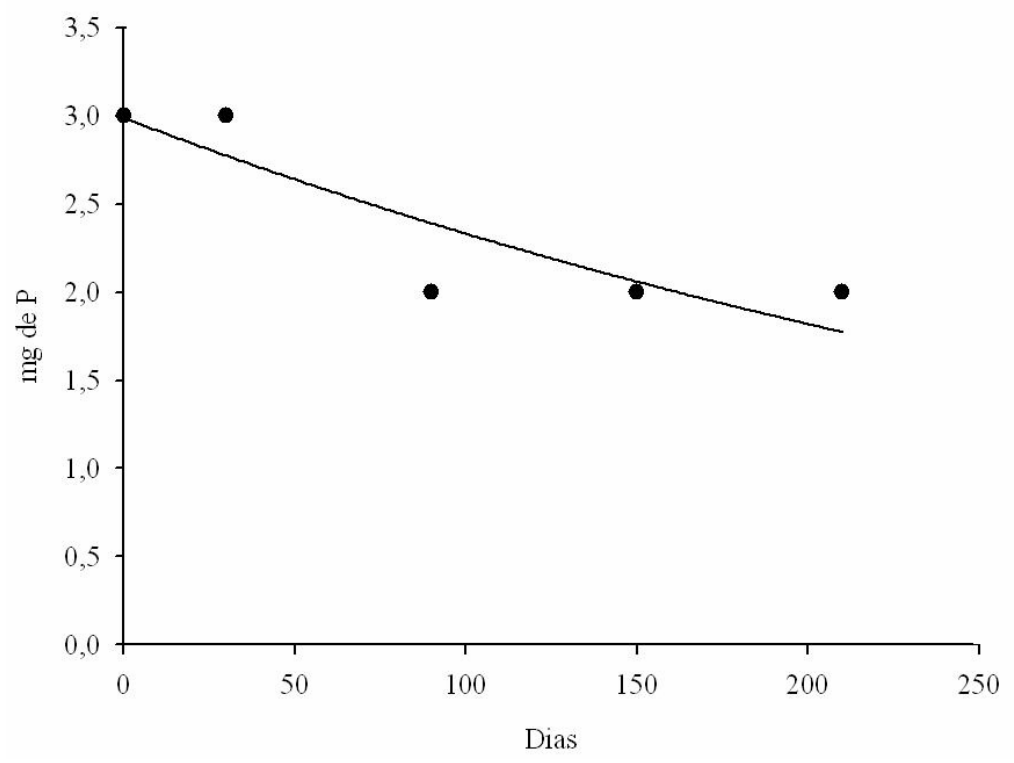

FIGURA 6: Liberação de fósforo na floresta atlântica da Ilha da Marambaia, RJ.

FIGURE 6: Phosphorus release in the Atlantic forest of the Island of Marambaia, RJ.

$\mathrm{O} \mathrm{K}$ foi o elemento cuja liberação ocorreu de maneira mais acentuada em relação aos outros analisados (Figura 7). Observa-se que grande parte do $\mathrm{K}$ foi perdida nos primeiros dias após a instalação das sacolas de decomposição. Para o K, verificou-se uma constante de decomposição (k) de $0,00563 \mathrm{~g} \mathrm{~g}^{-1}$ e um $\mathrm{T}^{1 / 2}$ de 12 dias. A intensa redução dos teores de $\mathrm{K}$ é decorrente das maiores perdas por lixiviação, já que esse elemento não faz parte de nenhum componente estrutural dos tecidos orgânicos, sendo facilmente mineralizável. A partir desse período, verifica-se uma maior estabilidade na liberação deste nutriente. Costa et al. (2005), estudando a decomposição e a liberação de nutrientes da serapilheira em povoamentos de Eucalyptus grandis na região norte fluminense, RJ, verificaram que o $\mathrm{K}$ foi rapidamente liberado da serapilheira, cerca de $50 \%$ até os 116 dias. Após esse período, o $\mathrm{K}$ remanescente manteve-se constante até o final do período de decomposição, conforme modelo exponencial ajustado.

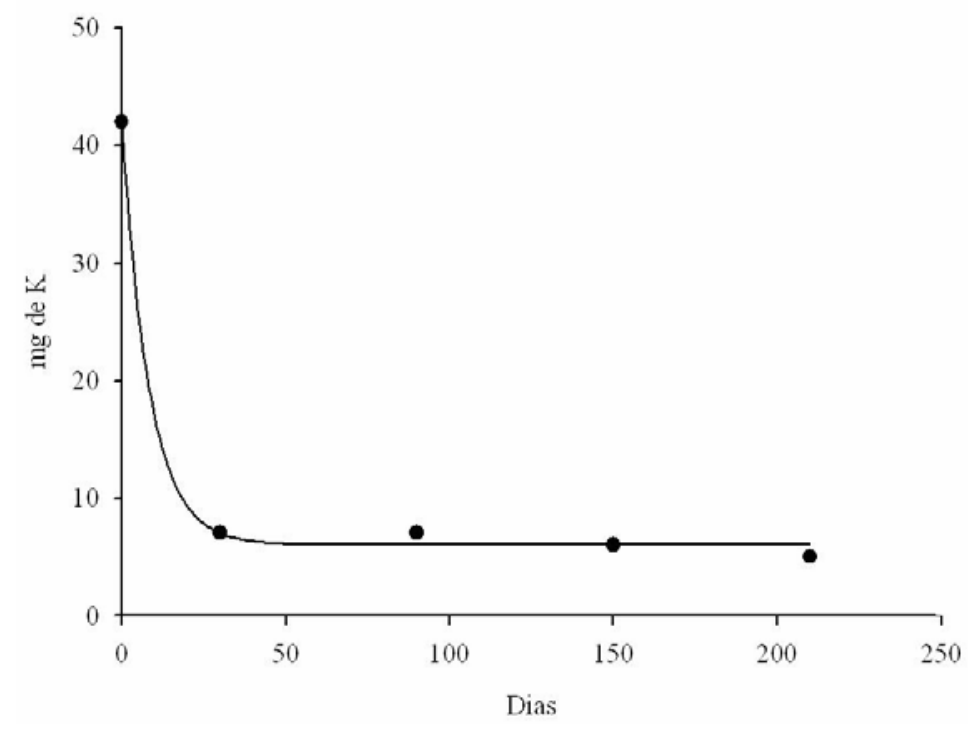

FIGURA 7: Liberação de potássio na floresta atlântica da Ilha da Marambaia, RJ.

FIGURE 7: Potassium release in the Atlantic forest of the Island of Marambaia, RJ. 


\section{Fertilidade do solo}

Os resultados das análises de fertilidade do solo são apresentados na Tabela 3. Com exceção do $\mathrm{P}$, os valores de carbono orgânico (C), $\mathrm{H}+\mathrm{Al}$ e dos nutrientes foram mais elevados na camada superficial diminuindo em profundidade. Os maiores valores em superfície são decorrentes do maior aporte de serapilheira. Acredita-se que a matéria orgânica seja a principal responsável pela retenção de cátions no solo estudado. Comportamento similar ao verificado neste estudo foi observado por Hay e Lacerda (1980) e Hay et al. (1981) em que autores verificaram correlações altas entre a matéria orgânica e a capacidade de troca catiônica para solos de restinga. Silva (1990) além da correlação da matéria orgânica e capacidade de troca catiônica nos solos da restinga da Ilha do Mel verificaram correlações significativas entre a matéria orgânica e os conteúdos de P, K, Mg e Ca. Pereira et al. (2005) estudando as propriedades químicas de solos sob Neoregelia cruenta (R.Grah) L.B. Smith na restinga da Marambaia (RJ) verificaram maiores valores de Ca, Mg e P nas áreas de ocorrência das bromélias em comparação às áreas desprovidas desta.

Verifica-se a diminuição dos valores de $\mathrm{pH}$ em profundidade, em decorrência do aumento dos teores de alumínio. Na camada de 10-20 cm de profundidade, observa-se percentagem de saturação por alumínio de 38\%. Britez et al. (1997) estudando solos de duas florestas da planície litorânea da Ilha do Mel (PR) verificaram forte acidez nos horizontes superficiais dos perfis do solo, o que promoveu uma alta saturação por alumínio tanto nos horizontes superficiais quanto em subsuperfície.

Os valores encontrados são ligeiramente superiores aos observados por Moraes et al. (1999) para a área de floresta Atlântica na Ilha do Cardoso (SP) indicando que a ciclagem de nutrientes nesse ecossistema é de grande importância para o desenvolvimento da vegetação.

TABELA 3: Análise química do solo da Floresta Atlântica da Ilha da Marambaia, RJ.

TABLE 3: Soil chemical analyses in the Atlantic Forest of the Island of Marambaia, RJ.

\begin{tabular}{|c|c|c|c|c|c|c|c|c|c|c|}
\hline \multirow{2}{*}{$\begin{array}{c}\text { Prof. } \\
\mathrm{cm}\end{array}$} & \multirow{2}{*}{$\mathrm{pH}$} & $\mathrm{C}$ & $\mathrm{N}$ & $\mathrm{P}$ & $\mathrm{K}$ & $\mathrm{Ca}$ & $\mathrm{Mg}$ & $\mathrm{Al}$ & $\mathrm{Na}$ & $\mathrm{H}+\mathrm{Al}$ \\
\hline & & \multicolumn{2}{|c|}{$\mathrm{g} \mathrm{kg}^{-1}$} & $\mathrm{mg} \mathrm{kg}^{-1}$ & \multicolumn{6}{|c|}{$\mathrm{cmol}_{\mathrm{c}} \mathrm{kg}^{-1}$} \\
\hline $0-5$ & 5,52 & 51,18 & 1,94 & 3,00 & 0,20 & 1,47 & 1,80 & 0,13 & 0,05 & 7,37 \\
\hline $5-10$ & 5,07 & 26,68 & 1,41 & 5,00 & 0,15 & 0,61 & 1,00 & 0,65 & 0,04 & 6,54 \\
\hline $10-20$ & 4,91 & 22,68 & 1,29 & 8,00 & 0,12 & 0,38 & 0,90 & 0,88 & 0,04 & 5,60 \\
\hline
\end{tabular}

\section{CONCLUSÃO}

A produção total de serapilheira durante o período observado foi de $7,9 \mathrm{Mg} \mathrm{ha}^{-1}$, em que a fração folhas foi a de maior contribuição. Os meses de maior e menor aporte foram novembro e junho respectivamente.

O retorno anual de nutrientes apresentou bastante similaridade com os verificados em outros estudos realizados em trechos de Mata Atlântica e áreas de restingas.

A taxa de decomposição do material formador de serapilheira foi de $40 \%$, nos 7 meses de observação, sendo o K o nutriente que apresentou a maior taxa de liberação.

O aporte de serapilheira e sua posterior decomposição são responsáveis pelos maiores teores de nutrientes na camada superficial do solo.

\section{AGRADECIMENTO}

Ao estudante de Ciências Biológicas Felipe Cito Nettsheim pela valorosa contribuição na identificação das famílias e espécies.

\section{REFERÊNCIAS BIBLIOGRÁFICAS}

AIDAR, M. P. M.; JOLY, C. A. Dinâmica da produção e decomposição da serapilheira do araribá (Centrolobium tomentosum Guill. Ex Benth. - Fabaceae) em uma mata ciliar, Rio Jacaré-Pepira, São Paulo. Revista Brasileira de Botânica, v. 26, n. 2, p. 193-202, 2003.

ALVAREZ-SÁNCHES, J; ENRÍQUEZ, R. B. Leaf decomposition in a Mexican tropical rain forest. Biotropica, v. 28 n. 4b, p. 657-667. 1996

BACKES, A.; PRATES, F. L.; VIOLA, M. Produção de serapilheira em floresta ombrófila mista, em São Francisco de Paula, Rio Grande do Sul, Brasil. Acta Botanica Brasilica v. 19, n. 1, p. 155-160. 2004

BARBOSA, J. H. C. Dinâmica da serrapilheira em estágios sucessionais de Florestas Atlântica (Reserva Biológica 
de Poços das Antas - RJ). 2000. 202p. Dissertação (Mestrado em Agronomia - Ciência do Solo) - Universidade Federal Rural do Rio de Janeiro, Seropédica, Rio de Janeiro, 2000.

BLOOMFIELD, J. VOGT, K. A.; VOGT, D. J. Decay rate and substrate quality of fine roots and foliage of two tropical tree species in the Luquillo. Experimental Forest, Puerto Rico. Plant and Soil, v. 150, p. 230-245. 1993.

BRAY, J. R.; GORHAM, E. Litter production in forests of the world. Advances in Ecological Research, London, v. 2, p. 101-157, 1964.

BRITEZ, R. M.; SANTOS FILHO, A.; REISSMANN, C. B.; SILVA, S. M.; ATHAYDE, S.F.; LIMA, R.X.; QUARDROS, R. M. B. Nutrientes no solo de duas florestas da planície litorânea da Ilha do Mel, Paranaguá - PR. Revista Brasileira de Ciência do Solo, v. 21, p. 625-634, 1997.

BRITEZ, R. M.; PIRES, L. A.; REISSMANN, C. B.; PAGANO, S.N.; SILVA, S. M.; ATHAYDE, S. F.; LIMA, R. X. 2005. Ciclagem de nutrientes na planície costeira. p. 145-168. In: M.C.M. Marques \& R.M. Britez (orgs.). História Natural e Conservação da Ilha do Mel. Curitiba, Editora da Universidade Federal do Paraná.

CARMO, M. R. B.; MORELlATO, L. P. C. Fenologia de árvores e arbustos das Matas Ciliares da Bacia do Rio Tibagi, Estado do Paraná, Brasil. In: RODRIGUES, R. R. \& LEITÃO FILHO, H. F (Eds). Matas ciliares: conservação e recuperação. São Paulo: EDUSP/FAPESP, Editora da USP/FAPESP, 2000, p. 125-141.

CONDE, M. M. S.; LIMA, H. R. P.; PEIXOTO, A. L. Aspectos florísticos e vegetacionais da Marambaia, Rio de Janeiro, Brasil. In: pp. 133-168. MENEZES, L. F. T. PEIXOTO, A. L.; ARAUJO; D. S. D. História Natural da Marambaia. Edur, Seropédica. 288p. 2005.

CORTEZ, J.; DEMARD, J.M.; BOTTNER, P.; MONROIZER, L.J. Decomposition of Mediterranean leaflitters: a microcosm experiment investigating relationships between decomposition rates and litter quality. Soil Biology and Biochemistry, Oxford, v. 28, p. 197-225. 1996.

COSTA, S. G.; GAMA-RODRIGUES, A. C; CUNHA, G. M. Decomposição e liberação de nutrientes da serapilheira foliar em povoamentos de Eucalyptus grandis no norte fluminense. Revista Árvore, v. 29, n 4, p. 563-570, 2005.

CIANCIARUSO, M.V.; PIRES, J. S. R.; DELITTI, W. B. C.; SILVA, E. F. L. P. Produção de serapilheira e decomposição do material foliar em um cerradão na Estação Ecológica de Jataí, município de Luiz Antônio, SP, Brasil. Acta Botanica Brasilica, v. 20, n. 1, p. 49-59. 2006.

CUEVAS, E.; MEDINA, E. Nutrients dynamics within Amazonian forest II: fine root growth, nutrient availability and leaf litter decomposition. Oecologia, v. 76, p. 222-235, 1988.

DELITTI, W. B. C. Estudos de ciclagem de nutrientes: instrumentos para a análise funcional de ecossistemas terrestres. Oecologia Brasiliensis, v. 1, p. 469-486. 1995.

DELITTI, W. B. C. Ciclagem de nutrientes minerais em matas ciliares. In: SIMPÓSIO SOBRE MATA CILIAR, 1989, São Paulo. Anais... São Paulo: Fundação Cargil, Secretaria do Meio Ambiente, Instituto de Botânica, 1989. p. 88-98.

DICKINSON, C. Decomposition of litter in soil. In: C. DICKINSON \& G. PUGH (Eds.). Biological of plant litter decomposition. Academic Press, USA. v. 2 p. 633-654. 1974.

EMBRAPA/CNPS. Manual de métodos de análise de solo. Rio de Janeiro, 212p. 1997.

FASSEBENDER, H. W. Modelos edafológicos de sistemas agroflorestais. $2^{\mathrm{a}}$ ed. Turrialba. Costa Rica: Centro Agronómico Tropical de Investigación y Enseñanza. 491p. 1993.

FIGUEIREDO-FILHO, A.; MORAES, G. F.; SCHAAF, L. C.; FIGUEIREDO, D. J. Avaliação estacional da deposição de serapilheira ombrófila mista localizada no sul do estado do Paraná. Ciência Florestal, v. 13, p. 11-18, 2003.

GAMA - RODRIGUES, A. C. da. Ciclagem de nutrientes por espécies florestais em povoamento puros e mistos, em solos de tabuleiros da Bahia, Brasil. 1997. 107p. Tese (Doutorado em Solos e Nutrição de Plantas) Universidade Federal de Viçosa, Viçosa, 1997.

GARAY, I. E.; KINDEL, A. Diversidade funcional em fragmentos de floresta atlântica: valor indicador das formas de húmus florestais. In: GARAY, I. E.; DIAS, B. Conservação da biodiversidade em ecossistemas tropicais: Avanços conceituais e revisão de novas metodologias de avaliação e monitoramento. Ed. Vozes, Petrópolis. 430p. 2001.

GONZÁLEZ, G.; SEASTEDT, T. R. Soil fauna and plant litter decomposition in tropical and subalpine forests. Ecology, v. 82, p. 955-964. 2001

HAY, J.D; LACERDA, L.D. Alterações nas características do solo após fixação de Neoregelia cruenta (R. Grah) L.Smith (Bromeliaceae), em um ecossistema de restinga. Ciência e Cultura, São Paulo, v. 32, p. 863-867, 1980.

HAY, J.D, LACERDA, L.D.; TAN, A. L. Soil-cation increase in a tropical sand dune ecosystem due to a terrestrial bromeliad. Ecology, Durham, v. 62, p. 1392-1395, 1981.

HERBOHN, J.L.; CONGDON, R.A. Ecosystem dynamics at disturbed and undisturbed sites in North Queensland wet tropical rain forest. II- Litterfall. Journal of Tropical Ecology. v. 9, p. 365-380. 1993 
IBGE. Manual Técnico da Vegetação do Brasil. Rio de Janeiro: Instituto Brasileiro de Geografia e Estatística (IBGE), 92p. 1992.

JACKSON, J. F. Seasonality of flowering of leaf-fall in a Brazilian Subtropical Lower Montane Moist Forest. Biotropica, v. 10, p. 38-42. 1978

JENSEN, V. Decomposition of angiosperm tree leaf litter. In. C. DICKINSON \& G. PUGH (Eds.). Biological of plant litter decomposition. Academic Press, USA. v. 2. p. 69- 104. 1974.

JORDAN. C. F. Nutrient cycling in tropical forest ecosystems. John Wiley \& Sons, Chichester, 190p. 1985.

KRAMMER, J. P.; KOZLOWSKI, T. Fisiologia das Árvores. Tradução de Antônio M. A. Magalhães. Fundação Calouste Gulbekian. 1960, 745p.

LA CARO, F. S.; RUDD, R. C. Leaf litter disappearance rate in Porto Rican Montane Rain Forest. Biotropica, v. 17, p. 269-276. 1985

LOPES, M. I. S.; DOMINGOS, M.; STRUFFALDI-DE VUONO, Y. Ciclagem de nutrientes minerais. In: SYLVESTRE, L. S.; ROSA, M. M. T. Manual metodológico para estudos botânicos na mata atlântica. EDUR UFRRJ, Seropédica, RJ, 2002, p. 72-102.

LOUZADA, M. A. P.; QUINTELA, M. F. S.; PENNA, L. P. S Estudo comparativo da produção de serapilheira em áreas de mata atlântica: a floresta secundária "antiga" e uma floresta secundária (capoeira). Oecologia Brasiliensis, V. 1, p. 61-74, 1995.

MARTINS, S. V.; RODRIGUES, R. R. Produção de serapilheira em clareiras de uma floresta estacional semidecídua no município de Campinas, SP. Revista Brasileira de Botânica, v. 22, p. 405-412. 1999.

MATTOS, C. C. L. V. Caracterização climática da restinga da Marambaia, RJ. In: p. 55- 66. L. F. T. Menezes, A. L. P., ARAUJO; D. S. D. História Natural da Marambaia. Edur, Seropédica. 288p. 2005

MEGURO, M., VINUEZA, G. N.; DELITTI, B. C. Ciclagem de nutrientes minerais na Mata Mesófila secundária - São Paulo. I - Produção e conteúdo de nutrientes minerais no folhedo. Boletim de Botânica da Universidade de São Paulo, v. 7, p. 11-31. 1979.

MENEZES, L. F. T. PEIXOTO, A. L.; ARAUJO; D. S. D. História natural da Marambaia. Edur, Seropédica. 288 p. 2005.

MORAES, R. M.; DELITTI, W. B. C.; STRUFFALDI, V. Y. Produção e conteúdo de nutrientes na serapilheira em duas florestas tropicais brasileiras. Revista Brasileira de Botânica, v. 22, n. 1, p. 09-16, 1999.

MORELLATO, L. P. C. Nutrient cycling in two Southeast Brazilian forest. Litterfall and litter standing crop. Journal of Tropical Ecology, v. 8, p. 205-215, 1992.

PAGANO, S.N. Produção de folhedo em mata mesófila semidecídua no município de Rio Claro, SP. Revista Brasileira de Biologia, v. 49, p. 633-639. 1989.

PAGANO, S. N.; DURIGAN, G. Aspectos de ciclagem de nutrientes em matas ciliares do Oeste do Estado de São Paulo, Brasil. In: RODRIGUES, R.R.; LEITÃO-FILHO, H. F. (Eds). Matas ciliares: conservação e recuperação. São Paulo: EDUSP/FAPESP, p. 109-123. 2000.

PEREIRA, M.G.; MENEZES, L. F.; SILVEIRA FILHO, T. B.; SILVA, A. N. Propriedades químicas de solos sob Neoregelia cruenta (R. Grah) L.B. Smith na restinga da Marambaia, RJ. Floresta e Ambiente, v. 12, n. 1, p. 70-73, 2005.

PIRES, L. A.; BRITEZ, R. M.; MARTEL, G.; PAGANO, S. N. Produção, acúmulo e decomposição da serapilheira em uma restinga da Ilha do Mel, Paranaguá, PR, Brasil. Acta Botanica Brasilica, v. 20, n. 1, p. 173-184. 2006

REZENDE, C. P.; CANTARUTTI, R. B.; BRAGA, J. M.; GOMIDE, J. A.; PEREIRA, J. M.; FERREIRA, E.; TARRÉ, R.; MACEDO, R.; ALVES, B. J. R.; URQUIAGA, S.; CADISCH, G.; GILLER, K. E.; BODDEY, R. M. Litter deposition and disappearance in Brachiaria pastures in Atlantic forest region of South Bahia, Brazil. Nutrient cycling in Agroecosystems, v. 54, p. 99-112. 1999.

SILVA, S. M. Composição florística e fitossociologica de um trecho de floresta de restinga na Ilha do Mel, município de Paranaguá, PR. 1990. 146p. Tese de Mestrado. Universidade Estadual de Campinas, Campinas, 1990.

STEVENSON, F. J. Humus chemistry - genesis, composition reactions. John Wiley \& Sons. New York, 1982. 443p. SWIFT, M. J.; HEAL, O. W.; ANDERSON, J. M. Decomposition in terrestrial ecosystems. $2^{\text {nd }}$ ed. University of California Press, Berkeley. 1979. 372p.

TEDESCO, J.; GIANELLO, C.; BISSANI, C.A.; BOHNEN, H.; VOLKWEISS, S.J. Análises de solo, planta e outros materiais. 2. Ed. Revista e ampliada. Porto Alegre, UFRGS, 174p. 1995.

THOMAS, R. J.; ASAKAWA, N. M. Decomposition of leaf litter from tropical forage grasses and legumes. Soil Biology and Biochemistry, v. 25, n. 10, p. 1351-1361. 1993 
TOLEDO, L. O.; PEREIRA, M. G. Dinâmica da deposição de serrapilheira em florestas secundárias do município de Pinheiral, RJ. Floresta e Ambiente, v. 11, n. 1, p. 39-46. 2004

VARJABEDIAN, R.; PAGANO, S.N. Produção e decomposição de folhedo em um trecho de Mata Atlântica de encosta no município do Guarujá. Acta Botanica Brasilica, v. 1, p.243-256. 1988

VILLELA, D. M.; PROCTOR, J. Leaf litter decomposition and monodominance in the Peltogyne Foresty of Maracá Island, Brazil. Biotropica, v. 34, n. 3, p. 334-347. 2002.

VITAL, A. R. T.; GUERRINI, I. A.; FRANKEN, W. K.; FONSECA, R. C. B. Produção de serapilheira e ciclagem de nutrientes de uma floresta estacional semidecidual em zona ripária. Viçosa, Revista Árvore, v. 28, n.6, p. 793-800. 2004.

WERNECK, M. S.; PEDRALLI, G.; GIESEKE, L. F. Produção de serapilheira em três trechos de uma floresta semidecídua com diferentes graus de perturbação na Estação Ecológica do Tripuí, Ouro Preto, MG. Revista Brasileira de Botânica, v. 24, n. 2, p. 195-198. 2001. 